\title{
Cardiovascular comorbidities in psoriasis (Review)
}

\author{
DACIANA ELENA BRANISTEANU ${ }^{1 *}$, ALIN CODRUT NICOLESCU ${ }^{2 *}$, DANIEL CONSTANTIN BRANISTEANU ${ }^{3 *}$, \\ CATALINA IOANA BRANISTEANU ${ }^{4}$, ANDREEA CORINA DRAGOI ${ }^{5}$, CAMELIA MARGARETA BOGDANICI $^{3}$, \\ MIHAELA PAULA TOADER ${ }^{6}$, ANDREEA LORENA TUCALIUC ${ }^{5}$, ANDREEA DIMITRIU ${ }^{7}$, \\ DORU DAOGARU ${ }^{8}$, RUXANDRA ANGELA PIRVULESCU $^{9,10}$ and ELENA ANDRESE PORUMB ${ }^{11}$
}

\footnotetext{
${ }^{1}$ Department of Dermatology, Faculty of Medicine ‘Grigore T. Popa' University of Medicine and Pharmacy, 700115 Iasi;

${ }^{2}$ Department of Dermatology, 'Roma' Medical Center for Diagnosis and Treatment, 011773 Bucharest; ${ }^{3}$ Department of Ophthalmology, Faculty of Medicine, 'Grigore T. Popa' University of Medicine and Pharmacy;

${ }^{4}$ Faculty of Medicine, 'Grigore T. Popa' University of Medicine and Pharmacy, 700115 Iasi;

${ }^{5}$ Department of Dermatology, Railway Clinical Hospital, 700506 Iasi; ${ }^{6}$ Department of Oral Dermatology,

Faculty of Dental Medicine, 'Grigore T. Popa' University of Medicine and Pharmacy, 700115 Iasi;

${ }^{7}$ Department of Dermatology, 'Arcadia' Hospitals and Medical Centers, 700620 Iasi;

${ }^{8}$ Department of Ophthalmology, 'Sf. Spiridon' Clinical Emergency County Hospital, 700111 Iasi;

${ }^{9}$ Department of Ophthalmology, Faculty of Medicine, 'Carol Davila' University of Medicine and Pharmacy, 050474 Bucharest; ${ }^{10}$ Ophthalmology Clinic, Emergency University Hospital, 050098 Bucharest;

${ }^{11}$ Department of Dermatology, 'Sf. Spiridon' Clinical Emergency County Hospital, 700111 Iasi, Romania
}

Received October 15, 2021; Accepted November 16, 2021

DOI: $10.3892 /$ etm.2021.11075

\begin{abstract}
Long considered a skin-limited condition, psoriasis is currently defined as a chronic, immune-mediated inflammatory disease, presenting, besides the skin changes, important systemic manifestations, the most common being: psoriatic arthritis, cardiovascular disease, metabolic syndrome, diabetes, inflammatory bowel disease and nonalcoholic steatohepatitis. It is a disease with a strong psycho-emotional and social impact, both through skin changes such as pruritic, scaly erythematous plaques, and through the association of comorbidities that influence morbidity and mortality. It has been shown that psoriasis is an independent cardiovascular risk factor, with patients developing ischemic heart disease/acute coronary syndrome, hypertension, peripheral arterial disease,
\end{abstract}

Correspondence to: Dr Andreea Corina Dragoi, Department of Dermatology, Railway Clinical Hospital, 1 Garabet Ibraileanu Street, 700506 Iasi, Romania

E-mail: d.corina94@yahoo.ro

Professor Camelia Margareta Bogdanici, Department of Ophthalmology, Faculty of Medicine, 'Grigore T. Popa' University of Medicine and Pharmacy, 16 Universitatii Street, 700115 Iasi, Romania

E-mail: camelia.bogdanici@umfiasi.ro

${ }^{*}$ Contributed equally

Key words: psoriasis comorbidities, cardiovascular disease, pathogenesis, screening, systemic therapy, biologic agents or stroke. The chronic inflammatory status of psoriasis and the production of specific cytokines may be the etiopathogenic link to atherosclerosis and cardiovascular disease. Biological therapy may affect atherosclerosis, leading to the arrest of the evolution or even regressing the changes in the atheromatous plaque. The aim of this review was to re-evaluate the current knowledge regarding the cardiovascular comorbidities associated with psoriasis for optimal management of the patients.

\section{Contents}

1. Introduction

2. Epidemiological data

3. Etiopathogenesis of psoriasis and cardiovascular disease

4. Cardiovascular risk factors

5. Cardiovascular risk in psoriasis: Sex differences

6. Screening methods for cardiovascular disease

7. Effects of systemic therapy

8. Conclusions

\section{Introduction}

Psoriasis is an immune-mediated papulo-squamous dermatosis, characterized by skin inflammation, epidermal hyperplasia with accelerated cell turnover and parakeratosis, dilated and tortuous vessels and a chronic inflammatory status responsible for intensely painful and destructive arthritis, increased cardiovascular risk and multiple comorbidities with significant psychosocial impact (1-4). From an etiopathogenic point of view, psoriasis is characterized by a polygenic 
predisposition, being triggered by a series of environmental factors such as trauma, infections, some drugs or stress, which activate the local native and adaptive immune system cells, producing proinflammatory cytokines $(1,5,6)$. It affects up to $3-5 \%$ of the general population, and about $20-30 \%$ of patients will develop psoriatic arthritis (4). In addition, those with moderate or severe form and early onset of the disease have an increased risk of metabolic syndrome and atherosclerosis, cardiovascular events being a morbidity and mortality factor of the disease (7). The approach of patients with psoriasis should include a complete clinical and paraclinical evaluation in order to identify and treat possible cardiovascular risk factors (8-10).

To collect recent reports of psoriasis cardiovascular comorbidities, a literature search was conducted using electronic databases Google Scholar, PubMed, Key Elsevier, UpToDate and Medscape for the terms 'psoriasis' in combination with 'cardiovascular disease', 'acute coronary syndrome', 'stroke', 'biologic therapies', 'pathogenesis', and 'treatment'. The present review includes case reports, case series, and literature review-type papers from the last 5 years. Based on 40 publications found in the literature, a concise report was compiled.

\section{Epidemiological data}

Due to the continuous development in the scientific field, psoriasis is currently known as an inflammatory disease with systemic involvement. Consequently, studies have been carried out on the association between psoriasis and comorbidities, among which cardiovascular disease is intensely discussed. The first study showed a twice as high risk of arterio-venous disease in patients with psoriasis. It is independent of the usual cardiovascular risk factors and is more common in severe forms of the disease in patients under 50 years of age (9). It affects both males and females, but it seems that men have a higher risk of having ischemic coronary heart disease (11), while women develop ischemic stroke (12). In the case of elderly psoriatic patients, the risk of vascular events was not higher compared to the control group, probably due to multiple risk factors present at this age (9).

\section{Etiopathogenesis of psoriasis and cardiovascular disease}

The link between psoriasis and cardiovascular disease has been studied by analyzing the responsible molecular mechanisms. These include common genetic factors and inflammatory pathways, adipokine secretion, insulin resistance, lipoproteins, angiogenesis, oxidative stress, and hypercoagulability (8).

Some genes associated with cardiovascular risk have been identified in patients with psoriasis, which may indicate a genetic predisposition. The association between psoriasis and cardiovascular disease can be partly explained by common inflammatory pathways (13). Chronic inflammation of the skin can lead to vascular and systemic inflammation, atherosclerosis and thrombosis. Patients with psoriasis have been shown to have increased vascular, subcutaneous, and hepatic inflammation (evaluated by PET/CT scan) compared to healthy controls. Furthermore, the $\mathrm{C}$-reactive protein (CRP), a systemic inflammatory marker, has been found to be elevated in patients with psoriasis. A previous meta-analysis found that the skin of psoriatic lesions showed increased gene expression associated with atherosclerosis signaling and fatty acid metabolism. The cytokine profiles of skin lesions of psoriasis and vascular lesions of atherosclerosis are very similar, showing an increased number of Th1 and Th17 lymphocytes (14). Similar to psoriasis, patients with ischemic heart disease have elevated levels of Th17-related cytokines [interleukin (IL)-17, IL-6 and IL-8] in their peripheral blood (6). Therefore, overexpression of Th17 cytokines in patients with psoriasis may mediate vascular inflammation and the development of atherosclerosis and cardiovascular comorbidities. However, the temporal relationship between systemic psoriatic inflammation and cardiovascular disease remains unclear. Systemic inflammation of psoriasis may lead to the development of cardiovascular disease or, alternatively, cardiovascular risk factors may cause immune dysfunction leading to psoriasis (15). Insulin resistance was more common in psoriatic patients vs. control, which could indicate a possible etiopathogenic link (16).

Both psoriatic skin lesions and atherosclerotic plaques are characterized by increased angiogenesis. The production of pro-angiogenic factors [including vascular endothelial growth factor (VEGF) and IL-8] in psoriasis plaques can lead to the development and progression of atherosclerosis. In addition, common pathways for signaling oxidative stress may underlie the association between psoriasis and atherosclerosis.

Homocysteine induces oxidative stress and it has been found that elevated plasma levels are associated with the development of atherosclerosis and cardiovascular disease. In patients with psoriasis, plasma homocysteine levels are elevated and folate levels decrease and correlate with the severity of the disease. This may contribute to the formation of atherosclerotic plaques in patients with psoriasis.

Patients with psoriasis have been shown to have increased platelet activation and aggregation, even elevated levels of the plasminogen activator inhibitor (PAI-1). These factors may lead to a state of hypercoagulability and an increased risk of thromboembolic events in these patients $(7-10,17)$.

\section{Cardiovascular risk factors}

According to studies, psoriasis is associated with an increased incidence of cardiovascular risk factors $(8,9)$.

Hypertension. There is an increased prevalence of high blood pressure in patients with moderate and severe forms of psoriasis, and uncontrolled forms of blood pressure are also correlated with the severity of the disease. At the same time, there is an increased incidence of psoriasis in hypertensive patients, probably in the context of antihypertensive treatment with beta-blockers, which constitute a trigger for dermatological manifestations.

Diabetes mellitus. Psoriasis promotes insulin resistance, so it could be a prediabetic disorder. At the same time, diabetic patients with psoriasis have a higher incidence of micro- and macrovascular complications of diabetes. Metformin treatment promotes weight loss and decreases the incidence of metabolic syndrome, ensures blood sugar control and thus it was observed to obtain a favorable appearance of the skin and reduce the risk of cardiovascular events. 
Dyslipidemia. An association with hypertriglyceridemia, hypercholesterolemia, with decreased HDL-cholesterol and increased LDL fraction has been found in both adult and pediatric psoriatic patients.

Obesity. Psoriatic patients usually have central obesity, and skin manifestations can be correlated with the degree of obesity (measured by body mass index-BMI). These findings have been observed in both adults and pediatric patients. The temporal relationship between psoriasis and obesity is under investigation. There is, on the one hand, an increase in the incidence of psoriasis in obese patients, but on the other hand, there is also an inverse relationship. The association of psoriasis-obesity significantly increases the incidence of cardiovascular disease.

Smoking and alcohol consumption. These are well known cardiovascular risk factors and have an increased prevalence in patients with psoriasis. A relationship between smoking and the severity of the disease has also been observed. At the same time, previous findings have shown an increased amount of Th17 lymphocytes in the peripheral blood in smokers, which could explain the increased risk of psoriasis in cigarette users (18).

\section{Cardiovascular risk in psoriasis: Sex differences}

The relationship between the presence of cardiovascular events and the patient's sex is well known, with women, especially young ones, having a lower cardiovascular risk compared to men of the same age. This is mainly explained by the hormonal status of the woman in the fertile period, estrogens having a potentially protective role on cardiovascular disease and metabolic syndrome (19). However, an inverse relationship has been observed in patients with psoriasis, with a higher prevalence of cardiovascular risk factors in young psoriatic women $(20,21)$.

A recent study conducted on a group of US hospitalized psoriatic patients, aged 20-35 years, showed that in the female group there were a higher number of cases with cardiovascular risk factors (obesity, dyslipidemia, smoking, diabetes mellitus), deep vein thrombosis and atherosclerotic cardiovascular disease, compared to the male group, which may show a higher vulnerability in women with psoriasis compared to men with psoriasis (21).

In a German study conducted on randomly selected patients, aged 45-75 years, there were considerable sex differences in cardiometabolic risk factors among those with psoriasis (20). Women with psoriasis showed higher prevalence of increased waist circumference, obesity, hypertriglyceridemia, hyperglycemia, diabetes, metabolic syndrome and intake of antihypertensive and antidiabetics. Unexpectedly, men had an inverse pattern. However, a relationship between sex and its effect on psoriasis cardiometabolic comorbidities is not yet clear. Further studies are needed in order to identify potential protective or maladaptive mechanisms on both sexes, as there may be a benefit from targeted screening of metabolic and cardiovascular disorders among psoriatic patients, with a special focus on women (20).

\section{Screening methods for cardiovascular disease}

In order to detect those patients with atherosclerosis risk, but asymptomatic, a useful tool can be imaging, by measuring the carotid intima-media thickness index (or in the femoral artery) and detecting atheroma plaque using ultrasound (14). Previous findings have shown that imaging can identify subclinical atherosclerosis in patients with psoriasis, which occurs more frequently in those with severe disease. Ultrasound is, therefore, an effective tool with minimal costs that can be used to diagnose atherosclerotic damage in psoriasis. In patients with psoriasis, there was an increased incidence of diastolic dysfunction of the left ventricle, with mitral regurgitation, which can be diagnosed using echocardiography (14).

A Japanese study on a group of patients with moderate to severe psoriasis showed the usefulness of the association between the ankle-brachial pressure index and coronary computed tomography angiography in diagnosing cardiovascular disorders (22). Given that coronary computed tomography angiography is an invasive method of diagnosis, difficult to achieve in all patients with psoriasis, the use of ankle-brachial pressure index, a quick and easy method of detecting vascular changes, is initially recommended (22).

NT-proBNP is a degradation product of BNP (type B natriuretic peptide), secreted by cardiomyocytes due to increased parietal tension. In patients with psoriasis, increased levels of NT-proBNP were observed compared to the control patients; thus, it can be used as a marker of cardiovascular disease $(8,9,14)$.

\section{Effects of systemic therapy}

The latest studies are focused on the results of systemic therapy used in the treatment of psoriasis on cardiovascular events, through their anti-inflammatory and anti-cytokine effect (23-25). The first-line systemic treatment used in psoriasis is Methotrexate, an inhibitor of dihydrofolate reductase, which may decrease the incidence of cardiovascular disease, myocardial infarction and stroke, especially when combined with folic acid. Recent findings on patients with rheumatoid arthritis and plaque psoriasis, who were treated with $20 \mathrm{mg} /$ week of Methotrexate, showed a decrease in the carotid intima-media thickness index compared with those receiving a lower dose or other disease-modifying antirheumatic drug $(14,26)$. However, Methotrexate may lead to kidney damage and decrease creatinine clearance, therefore the patients should be well hydrated and monitored, especially those with renal disease (27). Cyclosporine, an immunosuppressive agent, although effective on severe cutaneous manifestations, has some side effects associated with increased cardiovascular risk, such as hypertension, altered lipid profile, and renal dysfunction. Thus, it is advised that cyclosporine should be used for a short duration or even avoided when there is kidney disease (27).

Another option approved for psoriasis treatment is retinoids, such as Acitretin, which may retard atherosclerotic disease progression. However, it was associated with increases in cholesterol and triglycerides. Therefore, patients under retinoid treatment should have regular lipid screenings in order to prevent cardiometabolic diseases (27). 
Regarding biological therapy, based on the mechanism of action, it is divided into: $\mathrm{T}$ lymphocyte modulating agents (alefacept, efalizumab), TNF- $\alpha$ inhibitors (infliximab, adalimumab, etanercept), IL-12/IL-23 inhibitors (ustekinumab, briakinumab, guselkumab, risankizumab), and IL-17A inhibitors (ixekizumab, secukinumab) (1). The use of biological therapy significantly reduces inflammatory activity in psoriasis, this being demonstrated in various studies by measuring inflammation markers CRP, ESR and fibrinogen (28). It has recently been shown that biologic therapy prevents the formation and even reduces the growth of atheroma plaque $(23,29,30)$. Previous findings showed that patients treated with TNF- $\alpha$ inhibitors had a lower risk of developing major cardiovascular events than those treated with Methotrexate (31). The analysis of a group of US patients who underwent phototherapy vs. TNF- $\alpha$ inhibitors (adalimumab, etanercept, infliximab) showed that those receiving biologic treatment had significantly fewer cardiovascular events (myocardial infarction, stroke/transient ischemic attack, unstable angina) (32). Different atherosclerosis-associated biomarkers (E-selectin, IL-22, hsCRP) have been shown to drop significantly after a 12-week therapy with adalimumab (33). However, it is contraindicated in patients with moderate and severe heart failure (NYHA III/IV class) (14,34). TNF- $\alpha$ inhibitors improve endothelial function and decrease the risk of myocardial infarction (14). Etanercept is shown to improve insulin resistance and reduce the CRP, therefore having beneficial effect on vascular function (27). Different studies suggest that the use of ustekinumab, an IL-12/IL-23 inhibitor, may lower cardiovascular events (35). However, a recent study showed an association with acute coronary syndrome and stroke within the first 6 months of treatment initiation among patients at high cardiovascular risk, thus requiring caution when choosing this treatment for these patients (36). The incidence of atrial fibrillation and major cardiovascular events in patients diagnosed with psoriasis or psoriatic arthritis treated with TNF inhibitors vs. ustekinumab was examined and no significant differences were observed (37). A study on psoriatic patients undergoing secukinumab (IL-17A inhibitor) treatment, during a 52-week monitoring period reported one cardiovascular event in the placebo group vs. 15 arterial atherothrombotic events and one cardiovascular death in the secukinumab-treated group (38). Further comparative studies are needed between different biologic therapies on different types of psoriasis patients in order to establish a clear relationship and an optimal therapeutic approach. Current guidelines regarding the approach of psoriasis patients with cardiovascular comorbidities recommend choosing IL-17 inhibitors, IL-23 inhibitors and ustekinumab and avoiding the TNF inhibitors in NYHA class III/IV cardiac failure patients (an echocardiogram may be used in order to evaluate the ejection fraction; an ejection fraction $<50 \%$ contraindicates the use of TNFi) $(39,40)$.

\section{Conclusions}

Psoriasis, through its systemic inflammatory status, is an independent risk factor for atherogenesis and ischemic cardiovascular events. Common inflammatory pathways are the most suggestive and studied in this association and they represent the therapeutical target. There may be a link between the patient's sex and how it influences the occurrence of cardiovascular comorbidities in psoriatic patients. For early diagnosis and treatment, a series of imaging investigations can be used, even in asymptomatic patients. It is important to follow the future studies performed on patients undergoing biological therapy and its effects on cardiovascular comorbidities, in order to decide the optimal therapeutic management, adapted to each patient.

\section{Acknowledgements}

Not applicable.

\section{Funding}

Publishing funds were supported by the Association of Dermatologists from Moldova.

\section{Availability of data and materials}

All information provided in this review is documented by relevant references.

\section{Authors' contributions}

DEB, DCB and ACN contributed to the study design, participated in the entire review process, and prepared the manuscript. DD, CMB, MPT, ACD, RAP and CIB contributed to collecting the relevant literature, data analysis, and critical interpretation. ACD, ALT, EAP and AD conceived the concept of the review and modified the manuscript. All authors read and approved the final version of the manuscript for publication. Data authentication is not applicable.

\section{Ethics approval and consent to participate}

Not applicable.

\section{Patient consent for publication}

Not applicable.

\section{Authors' information}

Author CIB is a medical student at the 'Grigore T. Popa' University of Medicine and Pharmacy, Iasi, Romania.

\section{Competing interests}

All the authors declare that they have no competing interests.

\section{References}

1. Kang S: Psoriasis. In: Fitzpatrick's Dermatology. 9th edition. Vol 1, McGraw-Hill Education, New York, NY, pp457-494, 2019.

2. Porumb-Andrese E, Vâtă D, Postolică R, Stătescu L, Stătescu C, Grăjdeanu AI, Pătrașcu AI, Popescu IA and Solovastru LG: Association between personality type, affective distress profile and quality of life in patients with psoriasis vs. patients with cardiovascular disease. Exp Ther Med 18: 4967-4973, 2019. 
3. Batani A, Branisteanu DE, Ilie MA, Boda D, Ianosi S, Ianosi G and Caruntu C: Assessment of dermal papillary and microvascular parameters in psoriasis vulgaris using in vivo reflectance confocal microscopy. Exp Ther Med 15: 1241-1246, 2018.

4. Taranu T, Toader MP and Esanu I: The importance of dual dermatologic and psychiatric approach in psychocutaneous disorders. Rom J Oral Rehab 8: 18-27, 2016.

5. Van de Kerkhof PC and Nestlé FO: Psoriasis. In: Dermatology. Bolognia J, Schaffer J and Cerroni L (eds). 4th edition. Vol 1, Elsevier, Philadelphia, pp138-158, 2017.

6. Maranduca MA, Hurjui LL, Branisteanu DC, Serban DN, Branisteanu DE, Dima N and Serban IL: Skin-a vast organ with immunological function (Review). Exp Ther Med 20: 18-23, 2020.

7. Laws PM and Warren RB: Psoriasis and comorbidities In: Advances in Psoriasis. Weinberg JM and Lebwohl M (eds) Springer, Cham, pp363-397, 2021

8. Hu SC and Lan CE: Psoriasis and cardiovascular comorbidities: Focusing on severe vascular events, cardiovascular risk factors and implications for treatment. Int J Mol Sci 18: 2211, 2017.

9. Masson W, Lobo M and Molinero G: Psoriasis and cardiovascular risk: A comprehensive review. Adv Ther 37: 2017-2033, 2020.

10. Korman NJ: Management of psoriasis as a systemic disease: What is the evidence? Br J Dermatol 182: 840-848, 2020.

11. Gelfand JM, Neimann AL, Shin DB, Wang X, Margolis DJ and Troxel AB: Risk of myocardial infarction in patients with psoriasis. JAMA 296: 1735-1741, 2006.

12. Gelfand JM, Dommasch ED, Shin DB, Azfar RS, Kurd SK, Wang $\mathrm{X}$ and Troxel AB: The risk of stroke in patients with psoriasis. J Invest Dermatol 129: 2411-2418, 2009.

13. Ghazizadeh R, Shimizu H, Tosa $M$ and Ghazizadeh $M$ : Pathogenic mechanisms shared between psoriasis and cardiovascular disease. Int J Med Sci 7: 284-289, 2010.

14. Martinez-Moreno A, Ocampo-Candiani J and GarzaRodriguez V: Psoriasis and cardiovascular disease: A narrative review. Korean J Fam Med 42: 345-355, 2021.

15. Jindal S and Jindal N: Psoriasis and cardiovascular diseases: A literature review to determine the causal relationship. Cureus 10 e2195, 2018

16. Boehncke WH: Systemic inflammation and cardiovascular comorbidity in psoriasis patients: Causes and consequences. Front Immunol 9: 579, 2018

17. Gisondi P, Bellinato F, Girolomoni $G$ and Albanesi $C$ : Pathogenesis of chronic plaque psoriasis and its intersection with cardio-metabolic comorbidities. Front Pharmacol 11: 117, 2020.

18. Coumbe AG, Pritzker MR and Duprez DA: Cardiovascular risk and psoriasis: Beyond the traditional risk factors. Am J Med 127: 12-18, 2014.

19. Iorga A, Cunningham CM, Moazeni S, Ruffenach G, Umar S and Eghbali M: The protective role of estrogen and estrogen receptors in cardiovascular disease and the controversial use of estrogen therapy. Biol Sex Differ 8: 33, 2017.

20. Sondermann W, Djeudeu Deudjui DA, Körber A, Slomiany U, Brinker TJ, Erbel R and Moebus S: Psoriasis, cardiovascular risk factors and metabolic disorders: Sex-specific findings of a population-based study. J Eur Acad Dermatol Venereol 34: 779-786, 2020

21. Garshick MS, Vaidean G, Nikain CA, Chen Y, Smilowitz NR and Berger JS: Sex differences in the prevalence of vascular disease and risk factors in young hospitalized patients with psoriasis. Int J Womens Dermatol 5: 251-255, 2019.

22. Yamazaki F, Takehana K, Tanaka A, Son Y, Ozaki Y and Tanizaki H: Relationship between psoriasis and prevalence of cardiovascular disease in 88 Japanese patients. J Clin Med 10: 3640,2021

23. Valaiyaduppu Subas S, Mishra V, Busa V, Antony I, Marudhai S, Patel $\mathrm{M}$ and Cancarevic I: Cardiovascular involvement in psoriasis, diagnosing subclinical atherosclerosis, effects of biological and non-biological therapy: A literature review. Cureus 12: e11173, 2020

24. Mocanu M, Toader MP, Rezus E and Taranu T: Aspects concerning patient adherence to anti-TNF $\alpha$ therapy in psoriasis: A decade of clinical experience. Exp Ther Med 18: 4987-4992, 2019.

25. Branisteanu DE, Voicu CM, Cretu A, Dimitriu A, Luca MC and Salavastru CM: Adverse reactions of biological therapy for psoriasis. Rev Med Chir Soc Med Nat Iasi 119: 38-44, 2015.
26. Balanescu AR, Bojinca VC, Bojinca M, Donisan $\mathrm{T}$ and Balanescu SM: Cardiovascular effects of methotrexate in immune-mediated inflammatory diseases. Exp Ther Med 17: 1024-1029, 2019

27. Mikhaylov D, Hashim PW, Nektalova T and Goldenberg G: Systemic psoriasis therapies and comorbid disease in patients with psoriasis: A review of potential risks and benefits. J Clin Aesthet Dermatol 12: 46-54, 2019.

28. Grechin C, Solovastru LG, Vata D, Patrascu AI, Grajdeanu AI and Porumb-Andrese E: Inflammatory marker alteration in response to systemic therapies in psoriasis. Exp Ther Med 20: 42-46, 2020.

29. Elnabawi YA, Dey AK, Goyal A, Groenendyk JW, Chung JH, Belur AD, Rodante J, Harrington CL, Teague HL, Baumer Y, et al: Coronary artery plaque characteristics and treatment with biologic therapy in severe psoriasis: Results from a prospective observational study. Cardiovasc Res 115: 721-728, 2019.

30. Choi H, Uceda DE, Dey AK, Abdelrahman KM, Aksentijevich M, Rodante JA, Elnabawi YA, Reddy A, Keel A, Erb-Alvarez J, et al: Treatment of psoriasis with biologic therapy is associated with improvement of coronary artery plaque Lipid-rich necrotic core: Results from a prospective, observational study. Circ Cardiovasc Imaging 13: e011199, 2020.

31. Wu JJ, Guérin A, Sundaram M, Dea K, Cloutier M and Mulani P: Cardiovascular event risk assessment in psoriasis patients treated with tumor necrosis factor- $\alpha$ inhibitors versus methotrexate. J Am Acad Dermatol 76: 81-90, 2017.

32. Wu JJ, Sundaram M, Cloutier M, Gauthier-Loiselle M, Guérin A, Singh R and Ganguli A: The risk of cardiovascular events in psoriasis patients treated with tumor necrosis factor- $\alpha$ inhibitors versus phototherapy: An observational cohort study. J Am Acad Dermatol 79: 60-68, 2018.

33. Gkalpakiotis S, Arenbergerova M, Gkalpakioti P, Potockova J, Arenberger P and Kraml P: Impact of adalimumab treatment on cardiovascular risk biomarkers in psoriasis: Results of a pilot study. J Dermatol 44: 363-369, 2017.

34. Kovitwanichkanont T, Chong AH and Foley P: Beyond skin deep: Addressing comorbidities in psoriasis. Med J Aust 212: 528-534, 2020

35. Gelfand JM, Shin DB, Alavi A, Torigian DA, Werner T, Papadopoulos M, Takeshita J, Noe MH, Dey AK, Playford MP and Mehta NN: A Phase IV, randomized, double-blind, placebo-controlled crossover study of the effects of ustekinumab on vascular inflammation in psoriasis (the VIP-U Trial). J Invest Dermatol 140: 85-93.e2, 2020.

36. Poizeau F, Nowak E, Kerbrat S, Le Nautout B, Droitcourt C, Drici MD, Sbidian E, Guillot B, Bachelez H, Ait-Oufella H, et al: Association between early severe cardiovascular events and the initiation of treatment with the anti-interleukin $12 / 23 \mathrm{p} 40$ antibody Ustekinumab. JAMA Dermatol 156: 1208-1215, 2020

37. Lee MP, Desai RJ, Jin Y, Brill G, Ogdie A and Kim SC: Association of Ustekinumab vs. TNF inhibitor therapy with risk of atrial fibrillation and cardiovascular events in patients with psoriasis or psoriatic arthritis. JAMA Dermatol 155: 700-707, 2019.

38. Ait-Oufella H, Libby P and Tedgui A: Anticytokine immune therapy and atherothrombotic cardiovascular risk. Arterioscler Thromb Vasc Biol 39: 1510-1519, 2019.

39. Thatiparthi A, Martin A, Liu J, Egeberg A and Wu JJ: Biologic treatment algorithms for moderate-to-severe psoriasis with comorbid conditions and special populations: A review. Am J Clin Dermatol 22: 425-442, 2021.

40. Nast A, Smith C, Spuls PI, Avila Valle G, Bata-Csörgö Z, Boonen H, De Jong E, Garcia-Doval I, Gisondi P, KaurKnudsen D, et al: EuroGuiDerm Guideline on the systemic treatment of psoriasis vulgaris-Part 2: Specific clinical and comorbid situations. J Eur Acad Dermatol Venereol 35: 281-317, 2021

This work is licensed under a Creative Commons Attribution-NonCommercial-NoDerivatives 4.0 International (CC BY-NC-ND 4.0) License. 